\title{
Commentary \\ Ductal-lobar organisation of human breast tissue, its relevance in disease and a research objective: vector mapping of parenchyma in complete breasts (the Astley Cooper project)
}

James J Going

Division of Cancer Sciences and Molecular Pathology, University of Glasgow, Glasgow, UK

Corresponding author: James J Going, going@udcf.gla.ac.uk

Published: 17 July 2006

This article is online at http://breast-cancer-research.com/content/8/4/107

(c) 2006 BioMed Central Ltd

Breast Cancer Research 2006, 8:107 (doi:10.1186/bcr1527)

\begin{abstract}
A human breast has many lobes, which are highly variable in size and shape, each with one central duct, its peripheral branches and their associated glandular tissues. Realising the potential of new endoductal approaches to breast diagnosis and improving our understanding of breast cancer precursors will require greatly improved knowledge of this ductal-lobar anatomy and the distribution of cancer precursors within it. This architecture is very challenging to study in its entirety: whole-breast lobe mapping has only been achieved for two human breasts. Clearly, much more efficient techniques are required. Streamlined data capture and visualisation of breast parenchymal anatomy from thin and thick sections in a vector format would allow integrated mapping of whole-breast structure with conventional histology and molecular data. The 'Astley Cooper digital breast mapping project' is proposed as a name for this achievable research objective. Success would offer new insights into the development of breast cancer precursor lesions, allow testing of the important 'sick lobe' hypothesis, improve correlation with imaging studies and provide 'ground truth' for mathematical modelling of breast growth.
\end{abstract}

\section{Introduction}

Duct injection studies by Sir Astley Cooper [1] (Figure 1) in women who died during lactation showed that human breast tissue is organised into separate lobes, each composed of one central duct, its peripheral branches and their associated glandular tissues. Amazingly, contemporary awareness of this anatomy is actually inferior to Cooper's, because the breast lobes remain hard to visualise and his work is not sufficiently well known.

We owe it to women to advance beyond Cooper, because ignorance of breast structure compromises our ability to understand breast cancer origins and to evaluate the potential efficacy of newly developed intraductal approaches to the breast. The 'sick lobe' hypothesis [2], which proposes that entire lobes may be at increased risk of neoplastic transformation, would, if correct, be a powerful impetus towards lobe-directed diagnosis and treatment. However, new endoductal techniques like breast duct lavage and duct endoscopy [3] will require outstanding paradoxes of breast duct and glandular anatomy to be resolved and their relationships with early mammary neoplasia to be clarified if their diagnostic and therapeutic potential [4] are to be realised. Better knowledge of breast ductal-lobar anatomy is also required for understanding normal human breast development, relationships between cancer precursor lesions, the pathways by which pre-cancerous cells may spread within a breast, and whether there is any link between lobe size or structure and the probability of a cancer arising in that lobe. Unfortunately, conventional histology is uninformative on the scale of a whole breast and duct injection studies are frequently compromised by duct rupture, duct blockage and the great difficulty of injecting all ducts.

Strikingly, while only 5 to 9 nipple duct openings can be identified in life, and a similar number yield milk during lactation $[5,6]$, there is good evidence that there are many more ducts (median 27; range 11 to 48 , interquartile range 21 to 30 ) in the central nipple duct bundle [7]. Threedimensional reconstruction studies suggest that these ducts do not arise by duct branching in the nipple, and a distinction has been proposed between a minority of 'type $A$ ' nipple ducts, which open onto the skin surface, and a larger number of 'type B' nipple ducts, which arise in the dermis from skin appendages [7]. Cannulation of 'type A' ducts ought to be possible in the living breast, but 'type B' ducts may be inaccessible to this approach. This is obviously important for the ductal approach to the breast via the nipple.

There is also great variation in the extent and duct branching patterns of individual breast lobes. Three-quarters of all glandular tissue in one breast arose from only 6 of its 20 central ducts [7]. It is an attractive hypothesis that these six were 'type $A$ ' ducts and that, in general, a small number of type $A$ ducts will allow access to most of the breast parenchyma by duct lavage. There is, however, as yet no proof of this important conjecture. Many ducts that can be 
Figure 1

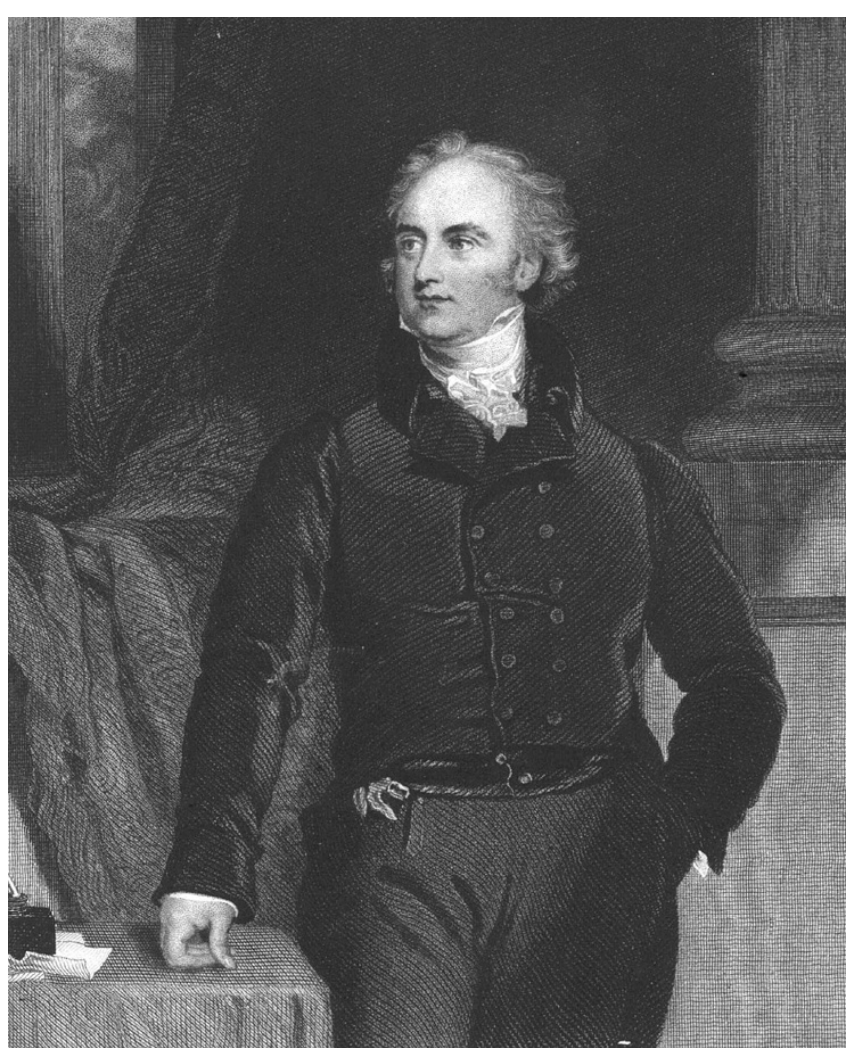

Sir Astley Paston Cooper. Steel engraving after the portrait by Sir Thomas Lawrence.

cannulated in vivo do indeed arborise widely in the breast; but others, up to several centimetres long, may end blindly without branching [8]. Whether any 'type B' ducts give rise to extensive breast lobes is unknown.

It is timely then to begin the work of filling in lacunae in our understanding of the lobar organisation of human breast and its physiological and pathological implications, and to establish methodologies facilitating further investigation of this important field. While challenging, this research agenda is entirely practicable. The 'Astley Cooper digital breast mapping project' is proposed as an appropriate name in tribute to Cooper's pioneering contributions in the field.

\section{Available methodologies}

Defining duct anatomy in the nipple and mapping duct branching in the mammary corpus present very different challenges. The duct bundle in the nipple is compact and crowded, so serial thin sections are a highly informative and appropriate investigational tool. As the ducts radiate from the nipple they rapidly become more widely spaced and can be traced through the much greater volume of the mammary corpus in serial thick sections, 1 to $2 \mathrm{~mm}$ thick, from which duct branching data can be extracted and digitally modelled [7-10]. The size of this challenge is implied by the fact that only two complete human breasts have been successfully studied in this manner $[7,9]$.

\section{Goals}

High-resolution three-dimensional digital modelling of ducts in the nipple

Three-dimensional reconstruction from conventional serial histological sections is prohibitively laborious, but episcopic fluorescence imaging, a recently developed technique [11], makes this much more feasible by capturing inherently registered images. These image stacks can be used to build three-dimensional digital models of duct structure $[7,10]$, using readily available software [12].

\section{Thick-section processing of entire breasts}

Thick-section processing of entire breasts, using either fixed or unfixed (chilled or frozen) tissue has a long history [13-16]. Whereas conventional histological sections are usually 3 to 4 microns in thickness, thick-section methodologies depend on the use of sections of one or two millimetres. It is possible to process a whole breast into thick sections. Ducts and lobules within thick sections can be stained and the sections subsequently cleared (rendered transparent) to allow the ducts and lobules to be observed in the slice. Some optimisation to maximise compatibility with subsequent molecular investigation is likely to be required.

\section{Developing data capture and visualisation of duct branching for all lobes in the same breasts}

Ducts can be seen entering, branching within and leaving a thick section, so it is possible to follow the ramifications of all ducts throughout a whole breast, but very laborious to do so by manual methods. A more efficient methodology is needed for such studies to be undertaken more widely. One practical obstacle is that although the points at which ducts leave a thick section are easily seen in a two-dimensional image, it is impossible to tell which side of the section the duct is leaving on, without studying adjacent sections. This makes it difficult to trace duct branching through an image stack. Note, however, that a branching system can be described by a series of straight line segments, each encoded as a pair of position vectors, one for the beginning and one for the end of the segment in a conventional Cartesian coordinate system. It is obvious that vectors describing all the duct elements in a thick section could be extracted from that section without any necessity to refer to adjacent sections. The plane of the section defines the $x-y$ plane. Some means to infer $z-$ coordinate measurements will be required. The most obvious approach is close-range photogrammetry of stereopairs, which could be collected on a flatbed scanner [17]. Assembling the final model will require registration of the points at which ducts leave one thick section and enter the next. There is no reason to suppose that this should be particularly challenging $[18,19]$. 
Linkage of nipple and whole breast duct models is analogous and should prevent no significant obstacles either.

\section{Mapping histological and molecular information relating to the same breasts onto the digital breast models}

Mapping histological and molecular information relating to the same breasts onto the digital breast models remains an important goal. An advantage of the vector-based mapping strategy proposed is that attributes such as histological descriptions and results of molecular or immunohistochemical analysis can be encoded and associated with the relevant vector objects (e.g., duct sections, lobules) in the three-dimensional breast parenchyma map for analysis and visualisation as appropriate. Ultimately, studies of ductalglandular structure in the nipple and mammary corpus need to be integrated with molecular investigations to maximise insights into the origins of human breast cancer.

\section{Research questions}

Is the distinction between 'type A' and 'type B' nipple ducts [7] confirmed? How many of each duct type are present in a typical breast? How much variation in the extent of different lobes is present within and between individual human breasts? What percentage of the mammary parenchyma is potentially accessible to endoductal diagnosis or treatment via 'type $A$ ' nipple ducts? Are there ductal anastamoses between different lobes? What is the relationship between lobe structure and cancer risk?

\section{Opportunities for investigating the anatomy of human breast}

Cancer mastectomies may not be optimal for whole-breast studies because of the necessity for immediate histological examination to determine treatment, but the Egan protocol [13] (chilling and sectioning a mastectomy breast prior to fixation) might allow thick-section study of even cancer-containing breasts without compromising their diagnostic evaluation. Routine histology of a normal-looking nipple is rarely of clinical value [20], so mastectomy nipples could be examined for research unless there was a particular reason not to. Prophylactic mastectomy for high cancer risk, in the presence of a strong family history or known mutation in a highpenetrance breast cancer related gene like BRCA1 or $B R C A 2$, is a different situation. Sometimes a cancer will have been detected, but in many cases imaging and examination will have detected none and in most of these breasts there is none [21]. Many will contain precursor lesions [22], however, and thick section examination followed by conventional histology could offer more comprehensive examination than ordinary routine pathology, provided it can be shown that diagnosis is not compromised. Women choosing prophylactic mastectomy may welcome a chance to increase our knowledge of the human breast in general and the high-risk breast in particular.

The opportunity to study complete normal breasts does not arise in life, but autopsy studies have considerable potential.
Visualisation of duct anatomy in the nipple and the mammary corpus in medical-legal autopsy breasts is proposed with appropriate consent.

The study of human material does present ethical and practical challenges, and it is worth noting that some of the approaches described in this commentary will also have potential application to animal models. Nevertheless, the challenges of studying human material cannot be shirked; there are sins of omission just as there are sins of commission. The challenge will be to prove that investigational procedures are not detrimental to the quality of pathological information extracted from surgical specimens, and for potential research participants to be accurately advised of the potential benefits and limitations of such studies, so that consent, when given, is truly informed.

\section{Conclusion}

Better knowledge of human ductal-lobar breast anatomy is essential for understanding the early origins of human breast cancer and rational design and evaluation of endoductal approaches to breast cancer diagnosis and treatment. Vector mapping of structural data from enhanced thick-section techniques would be applicable to autopsy and prophylactic mastectomy studies relevant to breast cancer origins, epidemiology, and breast imaging.

\section{Competing interests}

The author declares that they have no competing interests.

\section{References}

1. Cooper AP: On the Anatomy of the Breast. London: Longman, Orme, Green, Brown and Longmans; 1840.

2. Tot T: DCIS, cytokeratins, and the theory of the sick lobe. Virchows Archiv 2005, 447:1-8.

3. King BL, Love SM, Rochman S, Kim JA: The fourth international symposium on the intraductal approach to breast cancer, Santa Barbara, California, 10-13 March 2005. Breast Cancer Res 2005, 7:198-204.

4. Murata S, Kominsky SL, Vali M, Zhang Z, Garrett-Mayer E, Korz D, Huso D, Baker SD, Barber J, Jaffee E, et al.: Ductal access for prevention and therapy of mammary tumours. Cancer Res 2006, 66:638-645.

5. Love SM, Barsky SH: Anatomy of the nipple and breast ducts revisited. Cancer 2004, 101:1947-1957.

6. Ramsay DT, Kent JC, Hartmann RA, Hartmann PE: Anatomy of the lactating human breast redefined with ultrasound imaging. J Anatomy 2005, 206:525-534.

7. Going JJ, Moffat DF: Escaping from Flatland: clinical and biological aspects of human mammary duct anatomy in three dimensions. J Patho/ 2004, 203:538-544.

8. Moffat DF, Going JJ: Three dimensional anatomy of complete duct systems in human breast: pathological and developmental implications. J Clin Pathol 1996, 49:48-52.

9. Ohtake T, Kimijima I, Fukushima T, Yasuda M, Sekikawa K, Takenoshita S, Abe R: Computer-assisted complete threedimensional reconstruction of the mammary ductal/lobular systems: implications of ductal anastomoses for breast-conserving surgery. Cancer 2001, 91:2263-2272.

10. Going JJ, Mohun TM: Human breast duct anatomy, the 'sick lobe' hypothesis and intraductal approaches to breast cancer. Breast Cancer Res Treat 2006, 97:285-291.

11. Weninger WJ, Mohun T: Phenotyping transgenic embryos: a rapid 3-D screening method based on episcopic fluorescence image capturing. Nat Genetics 2002, 30:59-65. 
12. Fiala JC: Reconstruct: a free editor for serial section microscopy. J Microscopy 2005, 218:52-61.

13. Egan RL, Ellis JT, Powell RW: Team approach the study of diseases of the breast. Cancer 1969, 23:847-854.

14. Marcum RG, Wellings SR: Subgross pathology of the human breast: method and initial observations. J Natl Cancer Inst 1969, 42:115-121.

15. Wellings SR, Jensen HM, Marcum RG: An atlas of subgross pathology of the human breast with special reference to possible precancerous lesions. J Natl Cancer Inst 1975, 55:231273.

16. Jensen HM, Rice JR, Wellings SR: Preneoplastic lesions in the human breast. Science 1976, 191:295-297.

17. Schubert R: Using a flatbed scanner as a stereoscopic nearfield camera. IEEE Computer Graphics Applications 2000, 20: 38-45.

18. Griffin $\mathrm{P}$, Alexopoulos $\mathrm{C}$ : Point pattern matching using centroid bounding. IEEE Trans Systems, Man Cybernetics 1989, 19: 1274-1276.

19. Murtagh F: A new approach to point-pattern matching. Publications Astronomical Soc Pacific 1992, 104:301-307.

20. Gill MS, Staunton MJ: Routine sampling of the nipple in mastectomy specimens: pathology of limited clinical value? J Pathol 2005, 205s:8a.

21. Scott $\mathrm{Cl}$, lorgulescu DG, Thorne HJ, Henderson MA, Phillips KA: Clinical, pathological and genetic features of women at high familial risk of breast cancer undergoing prophylactic mastectomy. Clinical Genetics 2003, 64:111-121.

22. Kauff ND, Brogi E, Scheuer L, Pathak DR, Borgen PI, Hudis CA, Offit K, Robson ME: Epithelial lesions in prophylactic mastectomy specimens from women with BRCA mutations. Cancer 2003, 97:1601-1608 